TRANSACTIONS OF THE

AMERICAN MATHEMATICAL SOCIETY

Volume 358, Number 1, Pages 167-181

S 0002-9947(05)03820-1

Article electronically published on January 21, 2005

\title{
QUANTIFIER ELIMINATION FOR ALGEBRAIC $D$-GROUPS
}

\author{
PIOTR KOWALSKI AND ANAND PILLAY
}

\begin{abstract}
We prove that if $G$ is an algebraic $D$-group (in the sense of Buium over a differentially closed field $(K, \partial)$ of characteristic 0 , then the first order structure consisting of $G$ together with the algebraic $D$-subvarieties of $G, G \times$ $G, \ldots$, has quantifier-elimination. In other words, the projection on $G^{n}$ of a $D$-constructible subset of $G^{n+1}$ is $D$-constructible. Among the consequences is that any finite-dimensional differential algebraic group is interpretable in an algebraically closed field.
\end{abstract}

\section{INTRODUCTION}

This is the first of a series of three papers on algebraic groups with additional structure of a specific kind. In this first paper we will be concerned with algebraic varieties $X$ over a differentially closed field $(K, \partial)$ of characteristic zero. The additional structure on such $X$ will be an extension $\partial^{\prime}$ of $\partial$ to a derivation of the structure sheaf of $X$. Following Buium, we call $\left(X, \partial^{\prime}\right)$ an "algebraic $D$-variety" over $K$. $D$ here stands for $K[\partial]=\sum_{i \geq 0} K \partial^{i}$, the ring of linear differential operators generated by $K$ and $\partial$. If $C_{K}$ is the field of constants of $K$, then $K[\partial]$ will also be a $C_{K}$-algebra. The reason for the expression "algebraic $D$-variety" is that $\partial^{\prime}$ endows the structure sheaf of $X$ with the structure of a sheaf of $D$-modules, and conversely such a structure of sheaf of $D$-modules determines the extension $\partial^{\prime}$ of $\partial$. We stick with this formalism in the introduction but will adopt another equivalent presentation subsequently where precise definitions will be given.

If $\left(X, \partial^{\prime}\right),\left(Y, \partial^{\prime \prime}\right)$ are algebraic $D$-varieties over $K$, then $X \times Y$ is endowed naturally with an algebraic $D$-variety structure. Moreover, there are natural notions of a $D$-subvariety of $\left(X, \partial^{\prime}\right)$ and of a morphism between algebraic $D$-varieties. Here are some special cases:

(i) $X$ is defined over the constants $C_{K}$ of $K$. In this case $\delta^{\prime}$ is simply a vector field on $X$, and the $D$-subvarieties of $X$ which are defined over $C_{K}$ can be considered as "integral" subvarieties of $X$.

(ii) $X$ is again defined over $C_{K}$, and $\partial^{\prime}$ is obtained by tensoring the 0 -derivation of the structure sheaf of $X_{C_{K}}$ with $\partial$. In this case the $D$-subvarieties of $X$ (and likewise of $X \times X, \ldots)$ are precisely the algebraic subvarieties of $X$ which are defined over $C_{K}$.

Received by the editors January 5, 2004.

2000 Mathematics Subject Classification. Primary 32C38, 03C60.

The first author was supported by a postdoc under NSF Focused Research Grant DMS 0100979 and the Polish KBN grant 2 P03A 01824.

The second author was partially supported by NSF grants DMS 00-70179 and DMS 01-00979. 
We will call $\left(X, \partial^{\prime}\right)$ as in (ii), a trivial algebraic $D$-variety. We call $\left(X, \partial^{\prime}\right)$ isotrivial if it is isomorphic to a trivial $D$-variety.

We can and will consider an algebraic $D$-variety $\left(X, \partial^{\prime}\right)$ as a first order structure by identifying $X$ with its set of $K$-points, and adjoining predicates for $D$ subvarieties of $X, X \times X, \ldots$. With this convention, a motivating question behind the work in this paper is:

Question 1.1. Does every algebraic D-variety admit quantifier-elimination 1

This of course is the case when $\left(X, \partial^{\prime}\right)$ is isotrivial, as the algebraic $D$-subvarieties of $X^{n}$ will be precisely the algebraic subvarieties of $X^{n}$ defined over a suitable field of definition of $X$.

Let us now bring in "finite-dimensional differential algebraic varieties", which belong to Kolchin's "differential algebraic geometry". An affine differential algebraic variety is a subset $Z$ of $K^{n}$ defined by a finite number of differential polynomial equations $P\left(\bar{x}, \partial(\bar{x}), \partial^{2}(\bar{x}), \ldots\right)=0$. We call $Z$ finite-dimensional if the field of differential rational functions on $Z$ has finite transcendence degree over $K$. Equivalently, assuming $K$ to be $\omega$-saturated and $k$ to be a differential subfield of $K$ generated by the coefficients of the defining differential polynomials, there is a finite bound on the transcendence degree of $k\left(a, \partial(a), \partial^{2}(a), \ldots\right)$ over $k$, for $a \in Z$. Finite-dimensional differential algebraic varieties are built by piecing together such affine ones.

Buium B pointed out a close relation between finite-dimensional differential algebraic varieties and algebraic $D$-varieties. (See also P3.) Given an algebraic $D$-variety $\left(X, \partial^{\prime}\right)$, let $\left(X, \partial^{\prime}\right)^{\sharp}$ be the set of points $a \in X(K)$ such that the evaluation map from the local ring of $X$ at $a$ to $K$ is a $D$-module map. Then $X^{\sharp}$ is a finitedimensional differential algebraic variety, and birationally, every finite-dimensional differential algebraic variety arises this way. Moreover, the $\sharp$ map yields an equivalence of categories. A positive answer to Question 1 entails a rather stronger relationship between these two categories, as we now point out. For now let $L=L_{\left(X, \partial^{\prime}\right)}$ be the language of the first order structure on the algebraic $D$-variety $X$ mentioned earlier. Then $X^{\sharp}$ as a subset of $X(K)$ inherits an $L$-structure. One can show that all subsets of $\left(X^{\sharp}\right)^{n}$ definable in the differentially closed field $K$ are quantifier-free definable in the $L$-structure $X^{\sharp}$ (by quantifier-elimination in differentially closed fields). A positive answer to Question 1 is equivalent to $X^{\sharp}$ being an elementary substructure of $X$, as $L$-structures. A consequence would therefore be that the theory of any finite-dimensional differential algebraic variety, equipped with all its differential algebraic structure, is interpretable in the theory of algebraically closed fields of characteristic zero.

In this paper we answer Question 1 positively, and so draw all the above conclusions, in the special case when $\left(X, \partial^{\prime}\right)$ is an algebraic $D$-group. An algebraic $D$-group is an algebraic $D$-variety $\left(X, \partial^{\prime}\right)$ such that $X$ is equipped with the structure of an algebraic group, and $\partial^{\prime}$ respects this group structure. $X^{\sharp}$ is then a finite-dimensional differential algebraic group, and every finite-dimensional differential algebraic group arises this way. These objects (algebraic $D$-groups and finite-dimensional differential algebraic groups) were studied intensively and exhaustively in $[$ B. Our proofs make heavy use of the methods and results from [PZ.

\footnotetext{
${ }^{1}$ The second author has subsequently shown that the many-sorted structure $\mathcal{D}$ of all algebraic $D$-varieties does not have quantifier-elimination.
} 
In the current paper we often refer to variants or strengthenings of the so-called "socle theorem". This socle theorem originates in [H1, where it was proved that if $G$ is a commutative group of finite Morley rank satisfying a certain "rigidity" property, and $X$ is a definable subset of $G$ with finite stabilizer, then $X$ is internal to the family of strongly minimal sets in $G^{e q}$.

The second author would like to thank Ehud Hrushovski for many discussions in and around 1998. At this time the second author had observed that some nonisotrivial finite-dimensional differential algebraic groups, such as a certain differentially nonsplit extension of the additive group of the constants by the multiplicative group of the constants, are interpretable in algebraically closed fields. Hrushovski suggested the possibility that every finite-dimensional differential algebraic variety is "syntactically" interpretable in an algebraically closed field, via algebraic $D$-varieties. In fact, in [ $\mathrm{H} 2$, where Hrushovski uses the algebraic $D$-variety formalism, it is explicitly stated that Question 1 has a positive answer. However, we (Hrushovski and the second author) were unsuccessful in our attempts to verify this.

The second paper in this series will deal with the analogue of algebraic $D$-groups over fields of positive characteristic equipped with an iterative Hasse derivation. The third will deal with the analogue over fields equipped with an automorphism.

Finally, we are tempted to put forward a rather informal generalization of Zilber's conjecture: any structure of finite Morley rank "living in nature" is interpretable in an algebraically closed field. One case is: Let $X$ be a compact complex manifold equipped with all analytic subvarieties of $X, X \times X, \ldots$. Is $X$ is interpretable in an algebraically closed field?

In Section 2, we give precise definitions of algebraic $D$-varieties and groups, as well as extending results from $[\mathrm{PZ}]$ in this category. In Section 3, we prove the main results of the paper. In Section 4, we present some results from Section 2 in a general stable context, studying relative modularity for definable groups.

\section{Algebraic $D$-varieties}

Most of the definitions and some of the results of this section can be found in [P3], P4]. The general theory originates with Buium [B]. However, since in the forthcoming papers we plan to extend the results of the present paper to the difference and the Hasse case, we try to make this paper reasonably self-contained for future references.

We work over a saturated differentially closed field $(K, \delta)$ of characteristic 0 . Let $C$ denote the constant field. We assume familiarity with the model theory of differential fields as expounded in $[\mathbf{M}$, and in Section 4 we assume familiarity with stability theory. In this section, "definable" will usually mean "definable with parameters, in the structure $(K,+, \cdot, \partial)$ ", and $A$-definable means definable with parameters from $A$.

Recall that for any algebraic variety $V$ over $K$, we have a variety $\tau(V)$, a torsor of the tangent bundle to $V[\overline{\mathrm{PP}}$. If $V$ is defined over $C$, then $\tau(V)$ coincides with the tangent bundle. For a smooth affine variety $X \subset K^{n}$, we can also define $\tau(X)$ as the Zariski closure of the set $\left\{\left(x, \delta^{\times n}(x)\right): x \in X\right\}$. In any case $\tau(X)$ comes with a $(K, \delta)$-definable map $\delta_{X}: X \rightarrow \tau(X)$ which is a section of $\tau(V) \rightarrow V$.

Actually, $\tau$ is a functor and the maps $\tau(V) \rightarrow V$ and $\delta_{V}: V \rightarrow \tau(V)$ are natural, i.e., for any morphism $f: V \rightarrow W$, we get a morphism $\tau(f): \tau(V) \rightarrow \tau(W)$ such 
that the following diagrams commute:
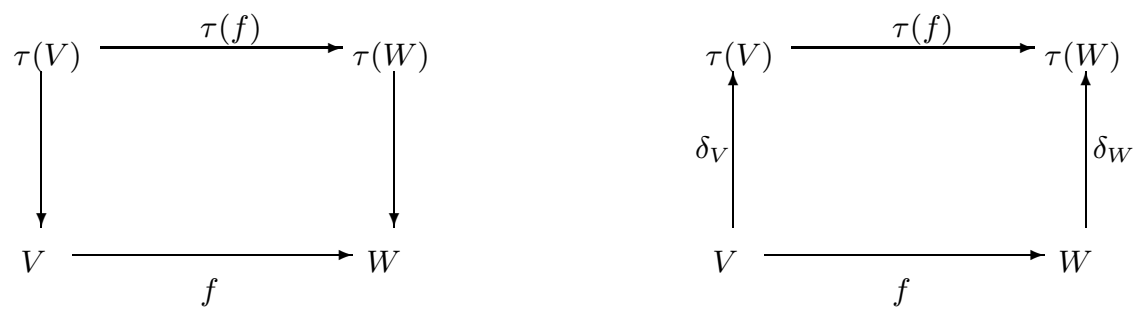

It is easy to see that $\tau$ preserves closed immersions. Therefore, if $W$ is a closed subvariety of $X$, we identify $\tau(W)$ with a subvariety of $\tau(X)$. Also, for any open subset $U \subset X$, the image of $\tau(U)$ in $\tau(X)$ coincides with the preimage of $U$ by $\tau(V) \rightarrow V$ and we identify $\tau(U)$ with its image in $\tau(X)$.

An algebraic $D$-variety (or just $D$-variety) is a pair $(X, s)$, where $X$ is a variety over $K$ and $s: X \rightarrow \tau(X)$ is a morphism which is also a section of $\tau(X) \rightarrow X$.

Note that if $X \subset K^{n}$ is affine, then giving $X$ a $D$-variety structure is equivalent to finding a derivation $\bar{\delta}$ of $K[X]$ (the coordinate ring of $X$ ) extending $\delta$ on $K$ (if $\bar{x}$ is the tuple of coordinate functions on $X$, then $\left.s=\left(\bar{x}, \bar{\delta}^{\times n}(\bar{x})\right)\right)$.

If $(X, s)$ is a $D$-variety, then for each open $U \subset X, s(U) \subset \tau(U)$, hence $(U, s \mid U)$ is a $D$-variety.

Putting this together we get:

Fact 2.1. For an algebraic variety $X$, giving a section $s: X \rightarrow \tau(X)$ is equivalent to giving a differential structure on a structure sheaf of $X$ extending the derivation on $K$.

If $X$ is affine, then it is enough to find such a derivation on its coordinate ring.

If $\left(V, s_{V}\right)$ and $\left(W, s_{W}\right)$ are $D$-varieties, then a $D$-morphism between them is a morphism $f: V \rightarrow W$ such that the following diagram is commutative:

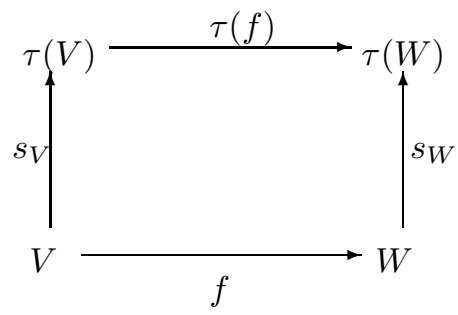

For affine $D$-varieties, $f$ being a $D$-morphism is equivalent to $f^{*}$ being a differential homomorphism of differential rings.

The notion of a $D$-rational map is clear as any open subset of a $D$-variety carries a $D$-variety structure.

We get a category of $D$-varieties with $D$-morphisms. This category obviously has products, since $\tau$ commutes with products. A $D$-algebraic group (or just a $D$-group) is a group object in this category, i.e., a $D$-variety $G$ such that $G$ is an algebraic group and the group operation is a $D$-map. In other words, $G$ is a $D$-group if $G$ is an algebraic group with a morphism $s: G \rightarrow \tau(G)$ which is a 
homomorphism of algebraic groups (since $\tau$ commutes with products, $\tau(G)$ has an algebraic group structure).

For a $D$-variety $(X, s)$, a closed subvariety $W$ is called $D$-subvariety, if $s(W) \subset$ $\tau(W)$. Again, it is obvious that for an affine $D$-variety $(X, s)$, a closed subvariety $W$ is a $D$-subvariety if and only if the ideal of $W$ is a differential ideal in $K[X]$.

If $X$ is defined over $C$, then a subvariety $W$ is a $D$-subvariety if and only if it is integral with respect to the vector field giving $X$ a $D$-variety structure.

Fact 2.2. (i) If $X$ is a $D$-variety, $V$ a closed subvariety, then $V$ is a $D$-subvariety if and only if for each open $U \subset X, \mathcal{I}_{V}(U)$ is a differential ideal of $\mathcal{O}_{X}(U)$.

(ii) An algebraic morphism between $D$-varieties $f: V \rightarrow W$ is a $D$-morphism if and only if for each open $U \subset W, f_{U}^{*}: \mathcal{O}_{W}(U) \rightarrow \mathcal{O}_{V}\left(f^{-1}(U)\right)$ is a homomorphism of differential rings.

(iii) Intersections and finite unions of D-subvarieties are D-subvarieties. Preimages of D-subvarieties by $D$-morphisms are $D$-subvarieties again.

Proof. (i) follows from the observation that for any open cover $\left(U_{i}\right)_{i}$ of $X, W$ is a $D$-subvariety of $X$ if and only if for any $i, W \cap U_{i}$ is a $\delta$-subvariety of $U_{i}$.

(ii) follows in a similar way as (i).

(iii) By (i) and (ii), we can assume that everything is affine. Then it is enough to see that radicals, sums, intersections and push-outs of differential ideals by differential homomorphisms are differential ideals again.

By 2.2(iii), we see that $D$-subvarieties are closed sets of a certain topology on $X$, which we call the $D$-Zariski topology. By 2.2(iii) again, $D$-maps are continuous in the $D$-Zariski topology.

For a $D$-variety $(X, s)$, the set of all $x \in X$ such that $s(x)=\delta_{X}(x)$ is denoted by $(X, s)^{\sharp}$ or by $X^{\sharp}$ when $s$ is understood. Therefore, $X^{\sharp}$ consists of closed points of $X$ in the $D$-Zariski topology and $X^{\sharp}$ is definable in $(K, \delta)$.

Fact 2.3. (i) A Zariski irreducible component of a D-subvariety is a D-subvariety.

(ii) If $X$ is a D-variety and $W$ is Zariski closed in $X$, then $W$ is a $D$-subvariety if and only if $W^{\sharp}$ is Zariski dense in $W$.

(iii) An algebraic morphism $f$ between $D$-varieties $\left(V, s_{V}\right),\left(W, s_{W}\right)$ is a $D$ morphism if and only if $f\left(V^{\sharp}\right) \subset W^{\sharp}$.

Proof. (i) This is Claim II(ii) on page 16 of $[\mathrm{P} 3$.

(ii) $\Rightarrow$ It is enough to show that $X^{\sharp}$ is Zariski dense in $X$ and this is [P3, 4.5].

$\Leftarrow \delta_{W}$ coincides with $s$ on $W^{\sharp}$. Therefore, $s\left(W^{\sharp}\right) \subset \tau(W)$. Since $W^{\sharp}$ is Zariski dense in $W, s(W) \subset \tau(W)$.

(iii) $\Rightarrow$ By the definitions.

$\Leftarrow$ For $x \in V^{\sharp}$ we have

$$
s_{W}(f(x))=\delta_{W}(f(x))=\tau(f)\left(\delta_{V}(x)\right)=\tau(f)\left(s_{V}(x)\right) .
$$

Therefore, (by (ii)), $s_{W} \circ f$ and $\tau(f) \circ s_{V}$ coincide on a Zariski dense subset of $V$, so they coincide on $V$.

By 2.3(iii), we get a $\sharp$-points functor from the category of $D$-varieties to the category of definable finite Morley rank sets in $(K, \delta)$ and definable maps.

The next proposition is Lemma 2.8 from Chapter 5 of $[\mathrm{B}]$ where the notion of a $\delta_{0}$-group (essentially a finite-dimensional differential algebraic group) is also defined. 
Proposition 2.4. The $\sharp$-points functor is an equivalence between the category of algebraic D-groups and the category of finite Morley rank groups definable in $(K, \delta)$.

Proof. By [P1], any finite Morley rank group $H$ definable in $(K, \delta)$ definably embeds into an algebraic group. In particular, $H$ is definably isomorphic to a $\delta_{0}$-group as in [B, 5.1.11]. By [B, 5.2.6] any $\delta_{0}$-group is definably isomorphic to $G^{\sharp}$ for a certain $D$-group $G$.

For $D$-groups $G_{1}, G_{2}$, any definable homomorphism between $G_{1}^{\sharp}$ and $G_{2}^{\sharp}$ extends to a definable in the pure field $K$ generically defined homomorphism between $G_{1}$ and $G_{2}$. Any generically defined homomorphism between algebraic groups (over a field of characteristic 0) extends to an everywhere defined homomorphism.

For an arbitrary subset $A$ of a $D$-variety $X, A^{\sharp}$ denotes $A \cap X^{\sharp}$. We conclude:

Lemma 2.5. Let $A, B, B_{i}, C_{i} \subset X ; X, Y$ be $D$-varieties and $f: X \rightarrow Y$ a $D$ morphism.

(i) If $A=\bigcup_{i} B_{i} \cap\left(X \backslash C_{i}\right)$, then $A^{\sharp}=\bigcup_{i} B_{i}^{\sharp} \cap\left(X^{\sharp} \backslash C_{i}^{\sharp}\right)$.

(ii) If $A, B$ are constructible in the D-Zariski topology, then $A^{\sharp}=B^{\sharp}$ implies $A=B$. In particular, $A^{\sharp}$ is Zariski dense in $A$.

(iii) If $A$ is constructible in the $D$-Zariski topology, then $f\left(A^{\sharp}\right)=f(A)^{\sharp}$.

Proof. (i) is obvious.

For (ii) it is enough to show the "in particular" part. By 2.3(i), we can assume that $A$ is irreducible in the subspace Zariski topology. Therefore, $A$ is of the form $V \cap U$ for a $D$-subvariety $V$ and Zariski open $U$. By 2.3(ii), $A^{\sharp}$ is Zariski dense in A.

(iii) By 2.3(iii), $f\left(A^{\sharp}\right) \subset f(A)^{\sharp}$. Take $a \in f(A)^{\sharp}$. Since $f$ is continuous in the $D$ Zariski topology, $f^{-1}(a) \cap A$ is constructible in the $D$-Zariski topology. Therefore, (by (ii)) $\left(f^{-1}(a) \cap A\right)^{\sharp}$ is non-empty.

It is easy to find counterexamples to 2.3(ii) and 2.5(ii) for non-Zariski-closed (respectively not constructible in the $D$-Zariski topology) subsets of a $D$-variety $X$. Take $U=X \backslash\{x\}$ for $x \notin X^{\sharp}$. Then $X^{\sharp}=U^{\sharp}$ but $X \neq U$ and $U^{\sharp}$ is Zariski dense in $U$, but $U$ is not constructible in the $D$-Zariski topology.

If $X$ is an algebraic variety defined over $C$, then it comes with a natural structure of a $D$-algebraic variety given by the 0 -section of the tangent bundle. We call such $D$-varieties trivial. We call a $D$-variety $D$-isotrivial, if it is isomorphic (as a $D$ variety) to a trivial $D$-variety. Note that the $D$-subvarieties of a trivial $D$-variety are exactly the subvarieties defined over $C$. So an algebraic $D$-group is $D$-isotrivial as an algebraic $D$-variety if and only if it is $D$-isotrivial as an algebraic $D$-group. Recall that a definable (in $(K, \partial)$ ) set $X$ is said to be $C$-internal if there is a definable function from some definable $Y \subseteq C^{n}$ onto $X$.

Fact 2.6. A $D$-group $G$ is $D$-isotrivial if and only if $G^{\sharp}$ is $C$-internal.

Proof. By 2.4, it is enough to show that any $C$-internal group definable in $(K, \delta)$ is definably isomorphic to the group of $C$-points of an algebraic group over $C$. This is obvious, since $C$ is stably embedded into $(K, \delta)$ and any definable group in $C$ is definably isomorphic to the group of $C$-points of an algebraic group over $C$. 
Fact 2.7. If $G$ is a $D$-group, $X$ an irreducible $D$-subvariety, and $H$ a $D$-subgroup, then:

(i) $\operatorname{Stab}(X)$ is a D-subvariety.

(ii) $G / H$ has a $D$-variety structure such that the quotient map is a D-morphism. If $H$ is normal, $G / H$ is a D-group.

(iii) $Z(G)$ is a $D$-subgroup.

Proof. (i) For $g \in G$ let $R_{g}, L_{g}: G \rightarrow G$ denotes right (resp. left) multiplication by $g$. Note that for $g \in G^{\sharp}, R_{g}$ and $L_{g}$ are $D$-morphisms. By 2.2(iii), any intersection of $R_{g}^{-1}(X)$ (for $g \in G^{\sharp}$ ) is a $D$-subvariety. We claim that

$$
\operatorname{Stab}(X)=\bigcap_{x \in X^{\sharp}} R_{x}^{-1}(X) .
$$

$\subseteq$ is obvious.

For $\supseteq$ take $g \in G$ such that $g X^{\sharp} \subseteq X$. Since $L_{g}^{-1}(X)$ is Zariski closed and $X^{\sharp}$ is Zariski dense in $X$ (2.3(ii)), $g \in \operatorname{Stab}(X)$.

(ii) We have a commutative diagram of algebraic groups:

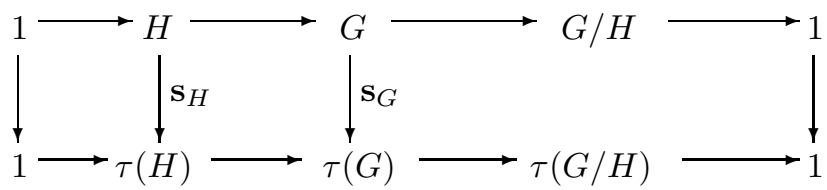

The upper row is exact (at $G / H$ in the pointed-sets sense).

The lower row is exact at $\tau(H)$ since $\tau$ preserves closed immersions. We want to prove that the whole lower row is exact. For a dominant map $X \rightarrow Y$, there exists $U \subset X$ which is open and dense such that $T_{x}(X) \rightarrow T_{f(y)} Y$ is surjective for $x \in U$ [Sh, 6.2, Lemma 2], where $T_{x} X$ is the tangent space to $X$ at $x$. We get the same conclusion after replacing $T_{x} X \rightarrow T_{f(y)} Y$ by $\tau_{x} X \rightarrow \tau_{f(y)} Y$, since $\tau$ is a torsor of the tangent space (see [PP]). In the case where $X \rightarrow Y$ is the quotient map $G \rightarrow G / H$, it is easy to see that we can take $U=G$, since group multiplication by $g$ induces isomorphisms $\tau_{x} G \cong \tau_{g x} G, \tau_{x / H} G / H \cong \tau_{g x / H} G / H$ commuting with the maps $\tau(G) \rightarrow G, \tau(G / H) \rightarrow G / H$.

Hence the whole lower row is exact, since $\tau$ takes a constant map to a constant map and $\operatorname{dim} \tau(G)=\operatorname{dim} \tau(H)+\operatorname{dim} \tau(G / H)$.

Let $s$ denote the composition of $\tau(G) \rightarrow \tau(G / H)$ with $s_{G}$. Since $s$ is constant on cosets of $H, s$ induces a morphism $s_{G / H}: G / H \rightarrow \tau(G / H)$ completing the above diagram and giving $G / H$ the required structure of a $D$-variety.

(iii) Note that by 2.2(iii) any intersection of $C(g)$ (for $g \in G^{\sharp}$ ) is a $D$-subgroup. We claim that

$$
Z(G)=\bigcap_{g \in G^{\sharp}} C(g) .
$$

$\subseteq$ is obvious.

For $\supseteq$ take $x \in G$ commuting with all elements from $G^{\sharp}$ and consider the map $f_{x}: G \rightarrow G, f_{x}(g)=x g x^{-1} g^{-1}$. For any $g \in G^{\sharp}, f_{x}(g)=1$, so $G^{\sharp} \subseteq f^{-1}(1)$. But $G^{\sharp}$ is Zariski dense in $G$ and $f^{-1}(1)$ is Zariski closed, so $f^{-1}(1)=G$. Therefore, $x \in Z(G)$. 
From now on we need the notion of a jet space. We refer the reader to [PZ] for background. We only mention that for an algebraic variety $X$ and $x \in X, J_{x}^{n}(X)$ denotes the $n$-th jet space to $X$ at $x$, which is a vector space over $K$. The first jet space coincides with the tangent space and if $X$ is a $D$-variety and $x \in X^{\sharp}$, then $J_{x}^{n}(X)$ has a $D$-variety structure.

Also for any finite-dimensional vector space $V$ over $K$, and $r \geq 1$ we have the $r$-grassmanian of $V$ denoted by $\operatorname{Gr}_{r}(V)$ which consists of $r$-dimensional linear subspaces of $V$. By specifying a basis of $V$, we can give $\operatorname{Gr}_{r}(V)$ the structure of an algebraic variety defined over $\mathbf{Q}$.

The next fact is a part of the proof of 2.1 from [P4].

Fact 2.8. Suppose $G$ is a D-group. For each $n$ there is a basis of $J_{e}^{n}(G)$ such that for any irreducible $D$-subvariety $X$, the Gauss map

$$
X \ni x \rightarrow J_{e}^{n}\left(x^{-1} X\right) \in \operatorname{Gr}_{r}\left(J_{e}^{n}(G)\right)
$$

(for appropriate $r$ ) is D-rational, where $\operatorname{Gr}_{r}\left(J_{e}^{n}(G)\right)$ has the trivial D-variety structure coming from the above basis.

Proof. Since $e \in G^{\sharp}, J_{e}^{r}(G)$ is a $D$-vector space. By 2.3(ii), we can find a basis of $J_{e}^{r}(G)$ in $J_{e}^{r}(G)^{\sharp}$. If we identify $J_{e}^{r}(G)$ with $K^{N}$ using the above basis, $J_{e}^{r}(G)^{\sharp}$ corresponds to $C^{N}$ and $\operatorname{Gr}_{r}\left(J_{e}^{n}(G)\right)(C)$ consists of subspaces of $K^{N}$ intersecting $C^{N}$ in a dense subset.

By 2.3(iii), it is enough to show that $J_{e}^{n}\left(x^{-1} X\right)$ has a dense intersection with $C^{N}$ for $x \in X^{\sharp}$. But for $x \in X^{\sharp}, x^{-1} X$ is a $D$-subvariety of $G$. Therefore, $J_{e}^{n}\left(x^{-1} X\right)$ becomes a $D$-linear subspace of $J_{e}^{r}(G)$ and $J_{e}^{n}\left(x^{-1} X\right) \cap C^{N}=J_{e}^{n}\left(x^{-1} X\right)^{\sharp}$ is Zariski dense in $J_{e}^{n}\left(x^{-1} X\right)$ by 2.3(ii) again.

As a corollary we get a variant of the "socle theorem" for $D$-groups. It was already proved (by the same methods) in [P4], and also can be obtained from "internality to the constants" and model theory as in [P2].

Corollary 2.9. If $G$ is a $D$-group and $X$ is an irreducible $D$-subvariety of $G$, then $X / \operatorname{Stab}(X)$ has a quotient D-variety structure and rationally $D$-embeds into a trivial D-variety (a grassmanian).

Proof. By 2.7(i), $\operatorname{Stab}(X)$ is a $D$-subgroup and by $2.7(\mathrm{ii}), G / \operatorname{Stab}(X)$ has a $D$ variety structure. As the Zariski topology of $G / \operatorname{Stab}(X)$ is the quotient one (see Bor, Chapter II, section 6]), by $2.5($ ii) and $2.3($ ii) $X / \operatorname{Stab}(X)$ is a $D$-subvariety of $G / \operatorname{Stab}(X)$.

As in [PZ, 2.4], for $n$ big enough the Gauss map as defined in Fact 2.8 is an embedding modulo $\operatorname{Stab}(X)$. Therefore, the rational $D$-map $X \rightarrow \operatorname{Gr}_{r}\left(J_{e}^{n}(G)\right)$ factors through a rational embedding $X / \operatorname{Stab}(X) \rightarrow \operatorname{Gr}\left(J_{e}^{n}(G)\right)$. The latter map is $D$-rational by 2.3 (iii).

We would like to use 2.9 to conclude that if $X$ contains the identity and generates $G$, then $G / \operatorname{Stab}(X)$ is $D$-isotrivial. However, $G / \operatorname{Stab}(X)$ is not a group anymore, so we cannot generate $G / \operatorname{Stab}(X)$ using $X / \operatorname{Stab}(X)$. But the next fact will yield an even better result. It was proved by Buium for centerless $G$ [B, Chapter $5,3.6]$.

Theorem 2.10. If $G$ is a connected algebraic D-group, then $G / Z(G)$ is $D$-isotrivial. 
Proof. For $g \in G$, let $H_{g}$ denotes the graph of conjugation by $g$. Consider the map (for appropriate $r$ )

$$
G / Z(G) \ni g / Z(G) \mapsto J_{e}^{n}\left(H_{g}\right) \in \operatorname{Gr}_{r}\left(J_{e}^{n}(G \times G)\right) .
$$

As in the proof of 2.8, the above map is $D$-rational if $\operatorname{Gr}_{r}\left(J_{e}^{n}(G \times G)\right)$ is given a trivial $D$-variety structure. For $n$ big enough, the above map is a rational embedding, so $(G / Z(G))^{\sharp}$ is $C$-internal. By $2.6, G / Z(G)$ is $D$-isotrivial.

Putting all this together we can prove a stronger version of the "socle theorem", which will be the main tool for the quantifier elimination result.

Theorem 2.11. Suppose that $G$ is a connected D-group, $X$ is an irreducible $D$ subvariety containing the identity and $X$ generates $G$. Then there is a normal $D$-subgroup $N$ of $G$ such that $N \subset \operatorname{Stab}(X)$ and $G / N$ is D-isotrivial.

Proof. Let $N=\operatorname{Stab}(X) \cap Z(G)$. We know that $X / \operatorname{Stab}(\mathrm{X})$ and $X / Z(G)$ rationally $D$-embed into trivial $D$-varieties. Hence $X / N$ rationally $D$-embeds into a trivial $D$-variety. Since $X / N$ generates $G / N,(G / N)^{\sharp}$ is $C$-internal. By 2.6, $G / N$ is $D$ isotrivial.

The appeal to internality and 2.6 at the end of the proofs of 2.10 and 2.11 can be avoided by the following whose proof does not use definability in differentially closed fields.

Fact 2.12. Suppose $G$ is a connected $D$-group, $X$ an irreducible $D$-subvariety containing the identity, generating $G$ and $D$-rationally equivalent to a trivial D-variety. Then $G$ is D-isotrivial.

Proof. Since the group operation is a $D$-map, we obtain a trivial $D$-variety $Y$ and a surjective $D$-rational map $f: Y \rightarrow G$. By shrinking $Y$ and passing to a cartesian power, we can assume that $f$ is everywhere defined.

Let $E$ be the equivalence relation on $Y$ given by $x E y \Leftrightarrow f(x)=f(y)$. As $E$ is $D$-closed in $Y \times Y$ (as the preimage of the diagonal by the $D$-morphism $f \times f: X^{2} \rightarrow G^{2}$ ), we get that $E$ is Zariski closed in $Y \times Y$ and defined over $C$.

By elimination of imaginaries in algebraically closed fields, there is an algebraic variety $Y^{\prime}$ defined over $C$ and a morphism $g$ defined over $C$ such that $f(x)=f(y) \Leftrightarrow$ $g(x)=g(y)$. Therefore, $g$ is a $D$-map of trivial varieties and $g$ factors through a $D$-rational $h: G \rightarrow Y^{\prime}$ which is an embedding.

Let $G^{\prime}=h(G)$. Since $Y^{\prime}$ is trivial, $G^{\prime}$ is a generically defined $C$-constructible group. Hence $G^{\prime}$ is definably isomorphic over $C$ to a $C$-constructible Zariski-dense subset of an algebraic group $G^{\prime \prime}$ defined over $C$. It is enough to check that the map $l: G \rightarrow G^{\prime \prime}$ is a $D$-morphism, when we equip $G^{\prime \prime}$ with the trivial $D$-variety structure.

But it is easy to see that on some open $U \subset G, l$ restricted to $U$ is a $D$-morphism, therefore $l$ has to be everywhere defined, so $l$ is a $D$-morphism itself.

The last two theorems also follow on the general ground from "the internality property" (see Section 4). In the last section, we give the general model-theoretical proofs, so the reader who prefers model-theoretical arguments than geometric ones, can just use the results of Section 4. 


\section{QUANTIFIER ELIMINATION AND ITS CONSEQUENCES}

We still work in a saturated differentially closed field $(K, \partial)$. Any algebraic $D$-variety $(X, s)$ may be considered as a structure in a natural language coming from $D$-subvarieties of cartesian powers of $X$ (in the same way as complex compact varieties are structures in the language coming from analytic subsets). That is, the language $L_{(X, s)}$ will consist of predicate symbols corresponding to $D$-subvarieties of $X, X \times X, \ldots$, and $X$ is an $L_{(X, s)}$-structure under the tautological interpretation. This structure is of course a reduct of $X$ equipped with all its algebraic subvarieties.

We are interested in whether the $L_{(X, s)}$-structure $X$ has elimination of quantifiers. Namely whether, if $Z \subset X^{n+1}$ is a finite Boolean combination of $D$ subvarieties, and $Z_{1}$ is the projection of $Z$ on $X^{n}$, then $Z_{1}$ is also a finite Boolean combination of $D$-subvarieties. Note that if $(X, s)$ is $D$-isotrivial, then this will be the case. We will use 2.11, to obtain quantifier elimination for algebraic $D$-groups.

Lemma 3.1. Suppose $V, V^{\prime}$ are D-varieties, $f: V \rightarrow V^{\prime}$ is a D-morphism, $X, Y \subset$ $V$ are proper D-subvarieties and $\operatorname{dim}$ denotes the algebraic dimension. Then we have:

(i) $\operatorname{dim}(X \cup Y)=\max (\operatorname{dim}(X), \operatorname{dim}(Y))$.

(ii) If $V$ is irreducible, then $\operatorname{dim}(X)<\operatorname{dim}(V)$.

(iii) If $X$ is irreducible, then

$$
\operatorname{dim}(X)=\operatorname{dim}(\overline{f(X)})+k
$$

where

$$
k=\min \left\{\operatorname{dim}\left(\pi^{-1}(a) \cap S\right): a \in \pi(S)^{\sharp}\right\} .
$$

Proof. (i) and (ii) are obvious and (iii) follows from an analogous statement in algebraic geometry [Sh, 6.3, Theorem 7 (ii)] and the fact that $\pi(S)^{\sharp}$ is Zariski dense in $\pi(S)(2.3($ ii) and $2.5($ iii $))$.

Theorem 3.2. Let $(G, s)$ be an algebraic D-group. Then the $L_{(G, s)}$-structure $G$ has quantifier-elimination.

Proof. Let $G, G_{0}$ be algebraic $D$-groups, let $\pi: G \rightarrow G_{0}$ be a $D$-homomorphism, and let $X \subset G$ be constructible in the $D$-Zariski topology. It suffices to prove that $\pi(X)=X_{0}$ is constructible in the $D$-Zariski topology (for quantifier elimination we take $G=G_{0} \times G_{0}$ and $\pi$ the projection map). As in [P2, 4.1], Lemma 3.1 implies that we can assume $X$ to be an irreducible $D$-subvariety. We can also assume that $1 \in X$ (after translation by an element from $X^{\sharp}$ ) and that $X$ generates $G$ (and so $G$ is connected also).

Let $N \subseteq \operatorname{Stab}(X)$ be a normal $D$-subgroup in $G$ satisfying the conclusion of 2.11 , let $\pi_{N}: G \rightarrow G / N$ be the quotient map and let

$$
X_{N}:=\pi_{N}(X)
$$

As in the proof of $2.9, X_{N}$ is a $D$-subvariety of $G / N$. Since $\pi(N)$ is Zariski closed, we conclude in the same way that $\pi(N)$ is a $D$-subgroup of $G_{0}$. By 2.7 (ii), we have 
a commutative diagram of $D$-groups:

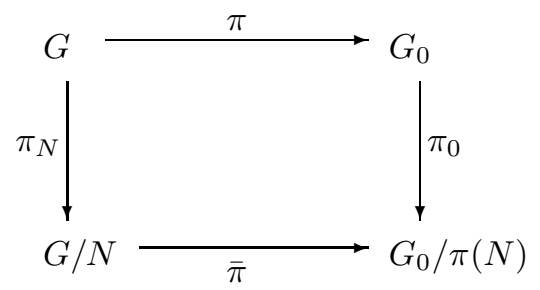

Let $X_{\pi(N)}:=\pi_{0}\left(X_{0}\right)=\bar{\pi}\left(X_{N}\right)$. We have $X_{0}=\pi_{0}^{-1}\left(X_{\pi(N)}\right)$. Since the preimage under a continuous map of a constructible set is constructible, it is enough to show that $X_{\pi(N)}$ is constructible in the $D$-Zariski topology.

By the choice of $N, G / N$ is $D$-isomorphic to a trivial $D$-group $H$. We obtain a $D$-homomorphism, $f: H \rightarrow G_{0} / \pi(N)$ and a commutative diagram of $D$-groups:

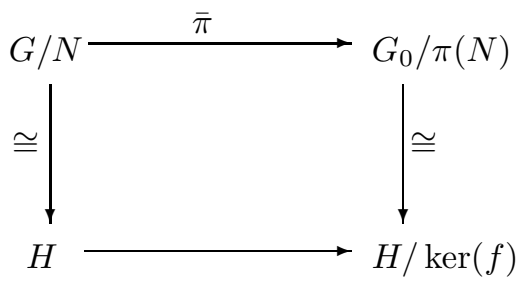

Since the theory of algebraically closed fields has elimination of imaginaries and quantifier elimination, the isomorphic image of $X_{N}$ in $H$ maps to a constructible set in $H / \operatorname{ker}(f)$. Therefore, $X_{\pi(N)}$ is $D$-constructible.

One might wonder whether it is possible to prove quantifier elimination for any $D$-algebraic variety $X$ just using quantifier elimination for $X^{\sharp}$ with the induced structure, which follows from quantifier elimination for $\mathrm{DCF}_{0}$. But what we would get is only that the $\sharp$-points of a projection of a $D$-subvariety coincide with a boolean combination of $\sharp$-points of certain $D$-subvarieties. But to get an equality on the level of boolean combinations of $D$-subvarieties we have to already know that a projection of a $D$-subvariety is constructible in the $D$-Zariski topology; see 2.5 (ii) and the counterexample after the proof of 2.5 .

In the same way as in the theory of compact complex spaces, we can consider a multi-sorted structure $\mathcal{M}$ of all $D$-varieties and a multi-sorted structure $\mathcal{G}$ of all $D$-groups. As a corollary of the proof we obtain:

Corollary 3.3. $\mathcal{G}$ has quantifier elimination.

Note that a $D$-algebraic variety satisfies all the axioms of a Zariski structure [Zi], P3], except that not all points are closed. We obtain the notion of a pre-Zariski structure by replacing the $T_{1}$-axiom by an axiom saying that inside any closed set closed points are dense and replacing axiom (iv)(b) from [P3] by 3.1(iii). It can be shown that for any pre-Zariski structure the set of its closed points with the induced topology and the induced notion of dimension is a Zariski structure. It was shown in [P3] that for any $D$-variety $X$, the set $X^{\sharp}$ is a Zariski structure. 
Given an algebraic $D$-variety $(X, s), X^{\sharp}$ as a subset of $X$ has an induced $L_{X, s^{-}}$ structure. Note that all subsets of the set $\left(X^{\sharp}\right)^{n}$ definable in $(K, \partial)$ are (quantifierfree) definable in the $L_{X, s}$-structure $X^{\sharp}$. Note also that every singleton in $X^{\sharp}$ is a $D$-subvariety of $X$, so is to all intents and purposes "named" by a constant of $L_{X, s}$. Here is our next main result.

Theorem 3.4. If $(G, s)$ is a D-algebraic group, then $G^{\sharp}$ is an $L_{G, s \text {-elementary }}$ substructure of $G$. More generally, if $\mathcal{G}^{\sharp}=\left\{G^{\sharp}: G \in \mathcal{G}\right\}$, then $\mathcal{G}^{\sharp} \prec \mathcal{G}$.

Proof. By Tarski-Vaught we must show that any $L_{G, s}$-formula $\phi(\bar{x})$ with parameters from $G^{\sharp}$ which has a solution in $G^{n}$ has a solution in $\left(G^{\sharp}\right)^{n}$. As the elements of $G^{\sharp}$ are named by constants, and $\operatorname{Th}(G)$ has quantifier-elimination in $L_{G, s}, \phi(\bar{x})$ defines a $D$-constructible subset $Z$ of $G^{n}$, which by assumption is nonempty. By Lemma 2.5 (ii), $Z^{\sharp}=Z \cap G^{\sharp}$ is nonempty. The proof is complete.

Among the consequences of Theorem 3.4 is that any group of finite Morley rank definable in $(K, \partial)$ is interpretable in a pure algebraically closed field.

Remark 3.5. Let $F$ be a differential subfield of $K$, and let $(G, s)$ be an algebraic $D$-group defined over $F$ (that is, both $G$ and $s$ are defined over $F$ ). Let $L_{(G, s), F} \subseteq$ $L_{(G, s)}$ be the (predicates) for algebraic $D$-subvarieties of various $G^{n}$ which are defined over $F$. Then $G$ has quantifier-elimination as an $L_{(G, s), F}$-structure, and every predicate in $L_{G, s}$-is definable from predicates in $L_{(G, s), F}$ with additional parameters.

Proof. By quantifier-elimination in differentially closed fields, the remark is true if we replace $G$ by $G^{\sharp}$. By 3.4 it is true of $G$ also.

We are unaware whether the following Corollary is known or interesting.

Corollary 3.6. Let $G$ be a connected algebraic group and $s$ a vector field which is also a homomorphism (from $G$ into the tangent bundle of $G$ ), all defined over an algebraically closed field $F$ of characteristic zero. Let $X$ be an "integral" subvariety of $G^{\times n}$-defined over $F$. Then the projection of $X$ to any $G^{\times m}$ is a Boolean combination of integral subvarieties (defined over $F$ ).

\section{Relative modularity in STABle theories}

The results from Section 2 can be extended to any stable structure satisfying a certain internality property.

In this section we work in a saturated multi-sorted stable structure $\mathcal{M}=\mathcal{M}^{\mathrm{eq}}$, $\mathcal{C}$ is an $\emptyset$-invariant class of types and the following property holds:

(*) For any $b, c \in \mathcal{M}, \operatorname{tp}(\mathrm{Cb}(\operatorname{stp}(c / b)) / c)$ is $\mathcal{C}$-internal.

For example, we can take as $\mathcal{M}$ finite-dimensional sets definable over some small differential field $F$ in a saturated differentially closed field $(K, \partial)$, equipped with all $F$-definable induced structure, and take $\mathcal{C}$ as the field of constants [PZ], or $\mathcal{M}$ could be a saturated elementary extension of the many-sorted structure of all compact complex spaces, and take $\mathcal{C}$ as the projective line $[\mathrm{P} 2$. It is shown in $[\mathrm{PZ}]$ that $\mathcal{M}$ can be also the class of very thin type-definable sets in $\mathrm{SCH}_{p, e}$ and $\mathcal{C}$ is then the field of absolute constants. In the latter case the structure may not be first-order.

If one is willing to include simple unstable examples, we can consider also the structure consisting of all finite SU-rank definable sets in a generic difference field 
of characteristic 0. $\mathcal{C}$ is the fixed field $[\mathrm{PZ}$. Zoe Chatzidakis $\mathrm{Ch}$ recently showed that the positive characteristic case also satisfies $(*$ ) (for $\mathcal{C}$, the collection of all fixed fields).

Recall that a stable theory is 1 -based or modular if for any $c, b, \mathrm{Cb}(\operatorname{stp}(c / b))$ is in $\operatorname{acl}(c)$. So $(*)$ holds in 1-based theories by taking $\mathcal{C}$ to be the class of algebraic types. Definable groups $G$ in 1-based theories (or even 1-based groups in stable theories) satisfy rather strong properties $[\mathrm{HP}$ :

(1) The Boolean algebra of definable subsets of $G$ is generated by cosets of definable subgroups.

(2) If $G$ is $A$-definable, then any connected definable subgroup $H$ of $G$ is definable over $\operatorname{acl}(\emptyset)$.

(3) $G$ is commutative-by-finite.

In this section, we will relativise these results.

The first property above is generalized by the following result, essentially proved in $[\mathrm{P} 2$.

Theorem 4.1. Suppose $G$ is a type-definable group in $\mathcal{M}, c \in G, \operatorname{tp}(c)$ is stationary and $H=\operatorname{Stab}(\operatorname{tp}(c))$ (left-stabilizer). Then $\operatorname{tp}(c / H)$ is $\mathcal{C}$-internal $(c / H$ regarded as an element of the right coset space $G / H)$.

Proof. Lemma 2.6 of $[\mathrm{P} 2$ says that if $a \in G$ is generic over $c$, then $c / H$ and $\mathrm{Cb}(\operatorname{stp}(a / c a))$ are interdefinable over $a$. So by the $(*)$-property, $\operatorname{tp}((c / H) / a)$ is $\mathcal{C}$-internal. As $c / H$ is independent from $a$ over $\emptyset, \operatorname{tp}(c / H)$ is $\mathcal{C}$-internal.

Note that dividing by a type-definable subgroup forces us to deal with hyperdefinable objects rather then type-definable in $\mathcal{M}^{\mathrm{eq}}(=\mathcal{M})$. But it does not present any troubles, since we can always think of an element of such a quotient as an inverse limit of elements from $\mathcal{M}^{\mathrm{eq}}$, since any type-definable group in a stable theory is an intersection of definable ones.

The above theorem suggests that all the non-modularity "comes from $\mathcal{C}$ ". We will see that this is actually the case by proving analogies of (2) and (3). We follow the lines from the proof of the 1-based case [P5].

Theorem 4.2. Suppose $G$ is a type-definable over $\emptyset$ group in $\mathcal{M}$, and that $H<G$ is a connected type-definable subgroup and $u$ is the canonical parameter of $H$. Then $\operatorname{tp}(u)$ is $\mathcal{C}$-internal.

Proof. Take $h \in H$ generic over $u$ and $g \in G$ generic over $u, h$.

Let $v$ be the canonical parameter of $g H$. Since $\operatorname{tp}(h / g, u)$ is the generic type of $H, \operatorname{tp}(g h / g, u)$ is the generic type of $g H$. Therefore, $v$ is interdefinable with $\mathrm{Cb}(\operatorname{stp}(g h / g, u))$. By the $(*)$-property, $\operatorname{tp}(v / g h)$ is $\mathcal{C}$-internal.

Since $H=(g H)^{-1} g H, \operatorname{tp}(u / g h)$ is $\mathcal{C}$-internal. By the choice of $(g, h), u$ is independent from $g h$, therefore $\operatorname{tp}(u)$ is $\mathcal{C}$-internal.

Theorem 4.3. Suppose $G$ is a connected type-definable group in $\mathcal{M}$. Then $G / Z(G)$ is $\mathcal{C}$-internal.

Proof. Let $g$ be generic in $G$ and let $H_{g}$ be the graph of conjugation by $g$. Then $g / Z(G)$ (considered as an element of $G / Z(G))$ is interdefinable with the canonical parameter of $H_{g}$. By the previous theorem, $\operatorname{tp}(g / Z(G))$ is $\mathcal{C}$-internal.

The last theorem generalizes 2.10 (modulo 2.6 and 2.7(iii)). 
In the analytic case, 4.3 says that in the category of complex Lie groups, if we take any extension of a complex torus by an algebraic group and divide it by its center, we get an algebraic group (i.e. a complex Lie group complex isomorphic to complex points of an algebraic group).

Now, we can prove what we call the "strong socle theorem". It generalizes 2.12 in the same way as 4.3 generalizes 2.11 .

Theorem 4.4. Let $G$ be a connected type-definable over $\emptyset$ group in $\mathcal{M}$. Suppose $p$ is a stationary type over $\emptyset$ realized in $G$. Suppose that $G$ is generated in finitely many steps by the set of realizations of $p$. (Namely there is $n$ such that every element of $G$ is a product of at most $n$ realizations of $p(x)$ or $p^{-1}(x)$.) Then there is $N$, a type-definable subgroup of $\operatorname{Stab}(p)$ which is normal in $G$ such that $G / N$ is $\mathcal{C}$-internal.

Proof. Let $H=\operatorname{Stab}(p)$. By $4.1, p / H$ is $\mathcal{C}$-internal, and by $4.3, p / Z(G)$ is $\mathcal{C}$ internal. As we have the following type-definable isomorphism of type-definable sets:

$$
G /(H \cap Z(G)) \cong G / H \times G / Z(G),
$$

we get that $p /(H \cap Z(G))$ is $\mathcal{C}$-internal. Since any subgroup of $Z(G)$ is normal in $G$, we can take $N=H \cap Z(G)$. Note that $p / N$ generates $G / N$ in finitely many steps, so $G / N$ is $\mathcal{C}$-internal.

Because of the examples mentioned after the definition of the $(*)$-property, one could ask the following:

Question 4.5. Suppose $\mathcal{M}$ is of finite U-rank (sort by sort) and $\mathcal{C}$ is the class of non-locally modular minimal types. Does $\mathcal{M}$ have the (*)-property?

Note that if we substitute analyzable in $\mathcal{C}$ for internal in $\mathcal{C}$ in $(*)$, then 4.5 has a positive answer ([ $[\mathrm{P} 6]$ and $[\mathrm{Ch}])$. So from the proof of 4.3 for example, we obtain the following result (which no longer assumes $(*))$ :

Theorem 4.6. Let $G$ be a group of finite Morley rank definable in some structure $\mathcal{M}$. Then $G / Z(G)$ is analyzable in the family of nonmodular strongly minimal sets in $\mathcal{M}$.

\section{REFERENCES}

[B] A. Buium, Differential Algebraic Groups of Finite Dimension, Springer-Verlag (1992). MR 1176753 (93i:12010)

[Bor] A. Borel, Linear Algebraic Groups, Second edition, Springer-Verlag, 1991. MR 1102012 (92d:20001)

[Ch] Z. Chatzidakis, A note on canonical bases and one-based types in supersimple theories, preprint 2002.

[H1] E. Hrushovski, The Mordell-Lang conjecture for function fields, J. Amer. Math. Soc. 9 (1996), 667-690. MR1333294 (97h:11154)

[H2] E. Hrushovski, Geometric Model Theory, Proceedings of ICM 1998, Vol. I, Documenta Math. 1998. MR1648035 (2000b:03120)

[HP] E. Hrushovski and A. Pillay, Weakly normal groups, in Logic Colloquium '85, NorthHolland, 1987. MR0895647 (88e:03051)

[M] D. Marker, Model theory of differential fields. In M. Messmer, D. Marker and A. Pillay, editors, The Model Theory of Fields, vol. 5 of Lecture Notes in Logic. Springer-Verlag, 1996. MR 1477154 (98m:03075)

[PP] D. Pierce and A. Pillay, A Note on the Axioms for Differentially Closed Fields of Characteristic Zero, Journal of Algebra 204 (1998), 108-115. MR.1623945 (99g:12006) 
[P1] A. Pillay, Some foundational questions concerning differential algebraic groups, Pacific Journal of Mathematics (1) 179 (1997), 179-200. MR1452531 (98g:12008)

[P2] A. Pillay, Model-theoretic consequences of a theorem of Campana and Fujiki, Fundamenta Mathematicae, 174 (2002), 187-192. MR1927236 (2003g:03057)

[P3] A. Pillay, Two remarks on differential fields, preprint, http://www.math.uiuc.edu/ People/pillay/remarks.difffields.pdf

[P4] A. Pillay, Mordell-Lang for function fields in characteristic 0, revisited, Compositio Math. 140 (2004), 64-68. MR2004123

[P5] A. Pillay, Geometric Stability Theory, Oxford University Press, 1996. MR 1429864 (98a:03049)

[P6] A. Pillay, Notes on analysability and canonical bases, preprint, http://www.math.uiuc.edu/ People/pillay/remark.zoe.pdf

[PZ] A. Pillay, M. Ziegler, Jet spaces of varieties over differential and difference fields, Selecta Math., New Ser. 9 (2003), 579-599. MR2031753 (2004m:12011)

[Sh] I. Shafarevich, Basic Algebraic Geometry 1, 2nd ed., Springer-Verlag, New York/Berlin 1994. MR:1328833 (95m:14001)

[Zi] B. Zilber, Model theory and algebraic geometry, Proceedings of 10th Easter Conference in Berlin, 1993, Humboldt Univ. of Berlin.

Department of Mathematics, Wroceaw University, Pl. Grunwaldzki 2/4, 50-384 WroceaW, POLAND

Department of Mathematics, University of Illinois at Urbana-Champaign, Urbana, ILLINOIS 61801 